\title{
Toxoplasma gondii infection in pet cats and their owners in northeastern China:an important public health concern
}

\author{
Xin-Tong Li, Lu Wang, Yuan Ding and Wu-Wen Sun*
}

\begin{abstract}
Background: Limited information about Toxoplasma gondii infection in pet cats and their owners is available in China.

Methods: In this study, blood samples were randomly collected from 306 pet cats and 397 corresponding pet owners in Jilin province, northeastern China. Sera from the pet cats and the pet owners were tested for anti-T. gondii antibodies using an modified agglutination test (MAT) and an enzyme-linked immunosorbent assay (ELISA), respectively. Moreover, the risk factors for T. gondii infection in pet cats and corresponding pet owners were explored.

Result: In total, 62 sera out of 306 examined pet cats (20.3\%) and 18.1\% (72/397) pet cat owners were seropositive for T. gondii, respectively. The results of statistical analysis showed that both pet cats and their owners from rural area had significantly higher T. gondii seroprevalence than those from urban area $(p<0.001)$. Moreover, owners of pet cas who have the knowledge of zoonotic protozoan diseases had a significantly lower $T$. gondii seroprevalence than those without the knowledge of zoonotic protozoan diseases $(p<0.001)$.

Conclusions: The present results revealed that the seroprevalence of T. gondii infection are widespread in pet cats and their owners in Jilin province, northeastern China. Residence area and understanding knowledge of zoonotic protozoan diseases are considered to be raleted to the T. gondii infection. Hence, it is necessary to highlight the dangers and protection methods of zoonotic protozoan diseases caused by pet cats, especially in rural area.
\end{abstract}

Keywords: Toxoplasma gondii, Pet cats, Owners, Seroprevalence, China

\section{Background}

With the rapid development of social economy and living standards, an increasing number of cats and dogs are raised as pets by many families in China [1]. Until now, there are over 50 million pet dogs and 40 million pet cats in China [2]. Considering the huge number of pets in China and the association with their owners, it is necessary for pet owners to know about the information of the zoonotic diseases transmitted by pets [3]. Toxoplasma

*Correspondence: sunwuwensci@163.com

College of Animal Science and Technology, Jilin Agricultural University,

Changchun, Jilin Province 130118, People's Republic of China gondii is an important zoonotic parasite belonging to Phylum Apicomplexa that is commonly found in warmblooded vertebrates, including humans and birds [4]. It has been calculated that nearly one-third of the global population has been infected by this parasite [5]. Cats, definitive hosts for this parasite, can discharge oocysts in their feces, resulting in soil contamination with oocysts [5]. Human can infect with this parasite through ingesting raw or undercooked meat containing T. gondii cysts, or through ingesting water or food contaminanted with T. gondii sporulated oocysts $[4,5]$.

Many studies have been conducted to explore the potential transmission situation of some zoonotic 
protozoan diseases between pets and humans all over the world $[4,6,7]$. However, limited information about $T$. gondii infection in pet owners in China can be obtained [8]. Thus, the present study was conducted with the aim to explore the T. gondii seroprevalence in pet cats and their owners for the first time in Jilin province, northeastern China.

\section{Results}

In this study, a total of 62 cat sera out of 306 examined pet cats $(20.3 \%)$ were seropositive for T. gondii with titers of 1:25 found in 15 pet cats, $1: 50$ in 9 pet cats, 1:100 in 9 pet cats, $1: 200$ in 7 pet cats, 1:400 in 10 pet cats, 1:800 in 9 pet cats, and $\geq 1: 1600$ in 3 pet cats (Table 1 ). Considering the species of pet cats, $T$. gondii seroprevalence was ranged from 0 in American Shorthair cat to 26.2\% in Chinese Lihua cat (Table 1). T. gondii seroprevalence in pet cats from Changchun, Jilin and Liaoyuan were 23.1, 19.8 and $16.9 \%$, respectively. In view of the age of pet cats, the cats were in the 2-3 year old age group have the highest $T$. gondii seroprevalence (22.1\%), followed by the $\leq 1$-year old age pet cats (19.8\%), and $>3$-year old age pet cats (17.6\%). Female pet cats (22.3\%) had a higher T. gondii seroprevalence than male pet cats (18.4\%). Moreover, pet cats from rural areas (30.4\%) had a significantly higher seroprevalence than those from urban areas (15.2\%) $(p=0.002)$.

The results of testing the $T$. gondii antibodies in the owners of pet cats showed that $18.1 \%(72 / 397)$ owners of pet cats were seropositive for $T$. gondii. Of these, T. gondii IgG and IgM antibodies were found in 16.9\% (67/397) and $1.3 \%(5 / 397)$ of the owners of pet cats, respectively. With a view to the age of owners of pet cats, T. gondii seroprevalence was ranged from $12.6 \%$ in the 31 - to 40 -year old age group to $24.0 \%$ in the $\leq 20$-year-old age group (Table 2). The seroprevalence of T. gondii infection in owners of pet cats from Changchun, Jilin, and Liaoyuan were $20.8,18.4$, and $13.9 \%$, respectively. Male owners of pet cats (19.4\%) had a litter higher seroprevalence than female owners (17.1\%). Moreover, owners of pet cats living in rural areas $(28.9 \%)$ had a significantly higher seroprevalence than those living in urban areas $(12.0 \%)(p<0.001)$. Owners of pet cats who had the habit of fecal harmless treatment (11.3\%), had a lower seroprevalence compared with those who did not have this habit (19.3\%), however the difference was not significant $(p>0.05)$. In addition, 39.8\% (158/397) owners of pet cats have the knowledge of zoonotic protozoan diseases, in this case, owners of pet cats who understood knowledge of zoonotic protozoan diseases $(10.1 \%)$ have a significantly lower seroprevalence than those who did not understand knowledge of zoonotic protozoan diseases $(23.4 \%)(p<0.001)$.

\section{Discussion}

In this study, we reported the evidence for the seroprevalence of T. gondii infection in pet cats and their owners in Jilin province, northeastern China for the first time. The proportion of $T$. gondii positive in the sera of pet cats and their owners were $20.3 \%(62 / 306)$ and $18.1 \%$ (72/397), respectively. The $T$. gondii seroprevalence in pet cats in this study (20.3\%) was a median of $20.3 \%$ T. gondii seroprevalence in cats including stray and pet cats reported by a systematic review and meta-analysis of the seroprevalence of $T$. gondii in cats in mainland China from 1995 to 2016 [9], but a litter lower than $21.67 \%$ T. gondii seroprevalence in pet cats in Shandong province, eastern China [8].

In China, toxoplasmosis is still an important public health problem because there is an increasing number of AIDS patients, and the number of people living with HIV and AIDS in China is nearly 1,000,000 [10]. It is well known that cats play a crucial role in the transmission of T. gondii $[4,5]$. In this case, pet cats could be a significantly potential cause of human toxoplasmosis because they frequently intimate contact with their owners. In China, owners of pet cats like to take their pets for a walk in the morning and evening time and the pet cats are free-roaming and might be exposed to $T$. gondii existed in the environment. After this, millions of environmentallyresistant oocysts might be excreted in cat feces, resulting in health risks to animals and humans $[5,11]$. Previous studies have showed that the presence of cats at home induces the risk of exposure to T. gondii $[12,13]$. Thus, it is very necessary to publicize the information of zoonotic protozoan diseases caused by pets to the public. Coincidentally, we found that the owners of pet cats who understood knowledge of zoonotic protozoan diseases (10.1\%) have a significantly lower seroprevalence than those who did not understand knowledge of zoonotic protozoan diseases $(23.4 \%) \quad(p<0.001)$. Therefore, publicity work should be taken to publicize the dangers and protection methods of zoonotic protozoan diseases caused by pets [1]. Furthermore, another known factor that contributes to the maintenance and dissemination of this disease, especially in rural environments, owners of pet cats consuming contaminated vegetables and fruits from home grown and poor sanitary conditions (untreated water). These factors increase the chances of getting infected more than owners of pet cats who live in cities. This is consistent with our research results that owners of pet cats living in rural areas (28.9\%) had a significantly higher seroprevalence than those living in urban areas $(12.0 \%)$ $(p<0.001)$.

Continuing evidence suggests that waterborne transmission of T. gondii to humans is common, through the spread of $T$. gondii oocysts by the overland runoff [14]. 


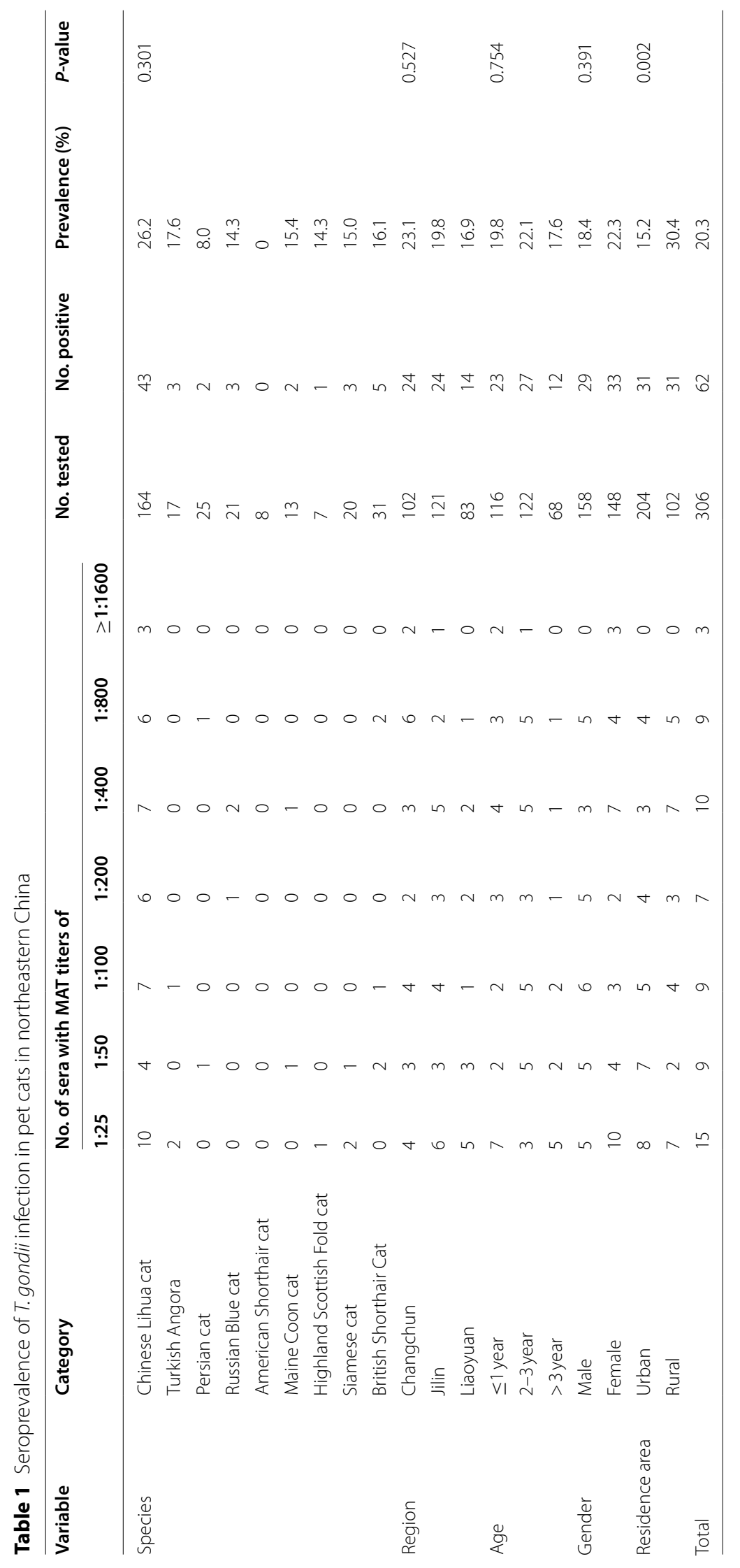


Table 2 Seroprevalence of T. gondii infection in the owners of pet cats in northeastern China

\begin{tabular}{|c|c|c|c|c|c|}
\hline Variable & Category & No. tested & No. positive & Prevalence (\%) & $P$-value \\
\hline \multirow[t]{5}{*}{ Age } & $\leq 20$ year & 50 & 12 & 24.0 & \multirow[t]{5}{*}{0.389} \\
\hline & 21-30year & 96 & 19 & 19.8 & \\
\hline & $31-40$ year & 119 & 15 & 12.6 & \\
\hline & 41-50year & 80 & 15 & 18.8 & \\
\hline & $>50$ year & 52 & 11 & 21.2 & \\
\hline \multirow[t]{3}{*}{ Region } & Changchun & 144 & 30 & 20.8 & \multirow[t]{3}{*}{0.376} \\
\hline & Jilin & 152 & 28 & 18.4 & \\
\hline & Liaoyuan & 101 & 14 & 13.9 & \\
\hline \multirow[t]{2}{*}{ Gender } & Male & 186 & 36 & 19.4 & \multirow[t]{2}{*}{0.554} \\
\hline & Female & 211 & 36 & 17.1 & \\
\hline \multirow[t]{2}{*}{ Residence area } & Urban & 259 & 31 & 12.0 & \multirow[t]{2}{*}{$<0.001$} \\
\hline & Rural & 142 & 41 & 28.9 & \\
\hline \multirow{2}{*}{$\begin{array}{l}\text { Understanding knowledge of Zoonotic } \\
\text { protozoan Diseases }\end{array}$} & Yes & 158 & 16 & 10.1 & \multirow[t]{2}{*}{$<0.001$} \\
\hline & No & 239 & 56 & 23.4 & \\
\hline \multirow[t]{2}{*}{ Fecal harmless treatment } & Yes & 62 & 7 & 11.3 & \multirow[t]{2}{*}{0.128} \\
\hline & No & 335 & 65 & 19.4 & \\
\hline Total & & 397 & 72 & 18.1 & \\
\hline
\end{tabular}

Thus, fecal harmless treatment of cats can cut down the environmental contamination with $T$. gondii oocysts existed in cat feces [15]. However, owners of pet cats who had the habit of fecal harmless treatment (11.3\%), had a lower seroprevalence compared with those without this habit (19.3\%) in the present study, however the difference was not significant $(p>0.05)$. Such a large quantity gap of sample-size between owners of pet cats who had the habit of fecal harmless treatment and those without the habit of fecal harmless treatment. Anyway, if we want to cut down the environmental contamination with $T$. gondii oocysts, we must hygienically dispose cat feces in the first place.

\section{Conclusions}

We firstly showed that the seroprevalence of $T$. gondii infection in pet cats and their owners is common in Jilin province, northeastern China. Thus, some control measures should be implemented to reduce $T$. gondii infection in pet cats, and the owner of pets, in the studied regions and elsewhere in China, such as publicity work and fecal harmless treatment.

\section{Materials and methods}

Northeast China is an important pet breeding and breeding base in China, and Jilin Province $\left(40^{\circ} 50^{\prime} \sim 46^{\circ} 19^{\prime}\right.$ $\left.\mathrm{N} ; 121^{\circ} 38^{\prime} \sim 131^{\circ} 19^{\prime} \mathrm{E}\right)$ is located in the central part of Northeast China. Due to geographical advantages and suitable environment, many residents here like to keep cats. The present study was approved by the Animal Ethics Committee of Jilin Agricultural University. From
January 2017 to October 2018, 306 pet cats were randomly selected from three regions (Changchun, Jilin, Liaoyuan) in Jilin province (Fig. 1). Questionnaires were provided to cat owners requesting data about each sampled animal. Collected data included information on the species, region, age, gender, and residence area of the pet cat, and their owner's age, region, gender, residence area, understanding knowledge of zoonotic protozoan diseases and how to deal with feces. Before blood collection of pet cats, a permission was obtained from each pet cat owner and then a local veterinary practitioner was employed to collect the blood samples from the medial saphenous vein of each pet cat. Moreover, we explained the purpose and process of this study to the pet owners and a permission was given to us from the pet owners. After that, nearly $2 \mathrm{ml}$ blood samples were got from the venous blood of each owner by a professional nurse. All blood samples were left about $4 \mathrm{~h}$ at $4{ }^{\circ} \mathrm{C}$ and then centrifugated at $1500 \times \mathrm{g}$ for $5-10 \mathrm{~min}$ to isolate the serum. After isolation, the serum were stored in Eppendorf tubes at $-20^{\circ} \mathrm{C}$ before the next step.

In this study, sera of pet cats were tested for anti$T$. gondii antibodies using a modified agglutination test (MAT) [16, 17]. Slightly, sera were added to the "U" bottom of 96-well microtiter plates, and diluted 2-fold starting from 1:25 to 1:1600. The sera was considered T. gondii-positive when the MAT titers $\geq 25$ [10]. Suspicious serum samples were re-tested. Moreover, positive and negative controls were included in all tests. To detect T. gondii IgG and IgM antibodies in sera of owners, commercially available enzymelinked 


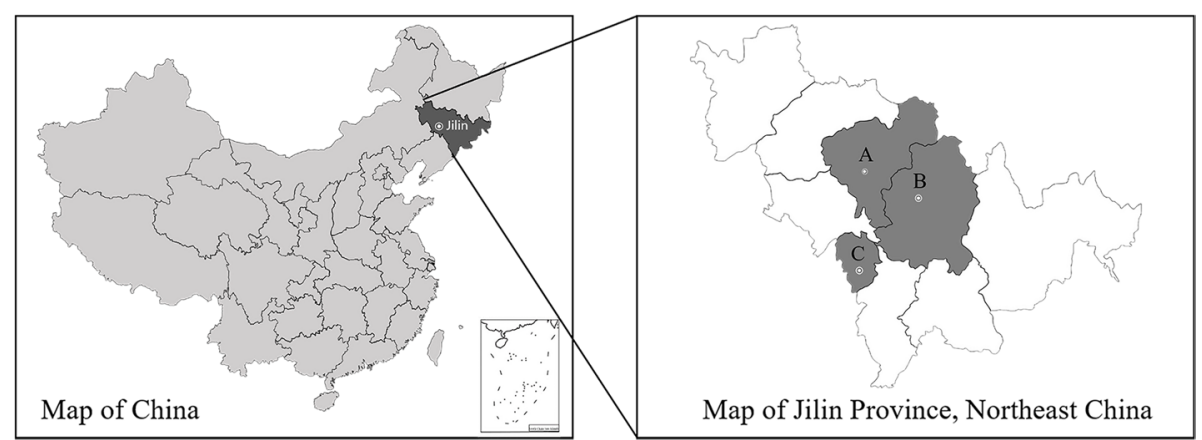

Fig. 1 Map showing three cities in Jilin province, northeastern China, where pet cats sampling was performed. A, Changchun; B, Jilin; C, Liaoyuan

immunosorbent assay (ELISA) kits (Haitai Co., Ltd., China) were used following the instructions of the manufacturer. All samples were run in duplicate. SAS version 9.1 was used to do the statistical analysis. $p$ value $<0.05$ was used to determine statistically significant by a Chi-square test.

\section{Abbreviations}

MAT: Modified Agglutination Test; ELISA: Enzyme-linked Immunosorbent Assay; SAS: Statistical Analysis System.

\section{Acknowledgements}

The authors would like to thank the study participants for their involvement in the study. We are grateful to the medical and veterinary staff for their help with the collection of blood samples.

\section{Authors' contributions}

WWS designed and coordinated the study. XTL collected the blood samples. $X T L, L W$, and YD performed laboratory analyses. XTL performed the statistical analyses and drafted the manuscript. All authors participated in data analysis and interpretation. All authors read and approved the final version of the manuscript.

\section{Funding}

Not applicable.

\section{Availability of data and materials}

The datasets included in the present study are available from the corresponding author upon request.

\section{Declarations}

\section{Ethics approval and consent to participate}

The study protocol was in accordance with the ethics guidelines of Jilin Agricultural University (JLAU) and the study was approved by the review board at JLAU, Changchun, China. The purpose of the study was explained to all study participants, and a written, informed consent was obtained prior to enrollment in the study. The sera were collected from the pets with a written agreement from the pet owners. Animals were handled in accordance with the requirements of the Animal Ethics Procedures and Guidelines of the People's Republic of China.

\section{Consent for publication}

Not applicable.

\section{Competing interests}

The authors declare that they have no competing interests.
Received: 17 April 2021 Accepted: 13 December 2021

Published online: 03 January 2022

\section{References}

1. Chen J, Xu MJ, Zhou DH, Song HQ, Wang CR, Zhu XQ. Canine and feline parasitic zoonoses in China. Parasit Vectors. 2012;5:152.

2. Chinese Pet Industry White Paper (Consumer report). 2020. p. 1-266.

3. Esch KJ, Petersen CA. Transmission and epidemiology of zoonotic protozoal diseases of companion animals. Clin Microbiol Rev. 2013;26(1):58-85.

4. Dubey JP. Toxoplasmosis of Animals and Humans. 2nd ed. Boca Raton: CRC Press; 2010. p. 313

5. Montoya JG, Liesenfeld O. Toxoplasmosis. Lancet. 2004;363:1965-76.

6. Zhang XC, Zhao N. Important pet-related parasitic zoonoses. Chin J Comp Med. 2010;20(Z1):65-70

7. Alho AM, Lima C, Colella V, Madeira de Carvalho L, Otranto D, Cardoso L. Awareness of zoonotic diseases and parasite control practices: a survey of dog and cat owners in Qatar. Parasit Vectors. 2018:11(1):133.

8. Cong W, Elsheikha HM, Zhou N, Peng P, Qin SY, Meng QF, et al. Prevalence of antibodies against toxoplasma gondii in pets and their owners in Shandong province, Eastern China. BMC Infect Dis. 2018;18(1):430.

9. Lopes AP, Oliveira AC, Granada S, Rodrigues FT, Papadopoulos E, Schallig H, et al. Antibodies to toxoplasma gondii and Leishmania spp. in domestic cats from Luanda, Angola. Vet Parasitol. 2017;239:15-8.

10. Györke A, Opsteegh M, Mircean V, lovu A, Cozma V. Toxoplasma gondii in Romanian household cats: evaluation of serological tests, epidemiology and risk factors. Prev Vet Med. 2011;102(4):321-8.

11. Ding H, Gao YM, Deng Y, Lamberton PH, Lu DB. A systematic review and meta-analysis of the seroprevalence of toxoplasma gondii in cats in mainland China. Parasit Vectors. 2017;10(1):27.

12. Wang Y, Huang KS. The size, spatial distribution and mortality characteristics of global AIDS population. Popul Soc. 2018;34:78-88.

13. Jones JL, Dargelas V, Roberts J, Press C, Remington JS, Montoya JG. Risk factors for toxoplasma gondii infection in the United States. Clin Infect Dis. 2009:49(6):878-84

14. Elsheikha HM. Congenital toxoplasmosis: priorities for further health promotion action. Public Health. 2008;122(4):335-53

15. Wang S, Zhou Y, Niu J, Xie Q, Xiao T, Chen Y, et al. Seroprevalence of toxoplasma gondii infection in domestic cats in Central China. Parasite. 2017;24:10.

16. Karanis P, Aldeyarbi HM, Mirhashemi ME, Khali KM. The impact of the waterborne transmission of toxoplasma gondii and analysis efforts for water detection: an overview and update. Environ Sci Pollut Res Int. 2013;20(1):86-99.

17. Torrey EF, Yolken RH. Toxoplasma oocysts as a public health problem. Trends Parasitol. 2013;29(8):380-4.

\section{Publisher's Note}

Springer Nature remains neutral with regard to jurisdictional claims in published maps and institutional affiliations. 